\title{
1 Foraging behavior across paths that vary in risk cues and frequency of occurrence
}

\author{
Emily K. Lessig', Peter Nonacs ${ }^{2 *}$ \\ ${ }^{1}$ Department of Ecology, Evolution, and Behavior, University of Texas at Austin, Austin, TX 78712 \\ ${ }^{2}$ Department of Ecology and Evolutionary Biology, University of California, Los Angeles, CA 90095 \\ E-mail address: pnonacs@biology.ucla.edu
}

\section{$\underline{\text { Abstract }}$}

14 environments to efficiently find and exploit food sources. These strategies become more

15 complicated when food and risk can be simultaneously present and when they differ in

16 predictability. For example, there may be multiple paths to reach a foraging site that vary in risk.

17 This study examines how colonies of Argentine ants (Linepithema humile) respond to such a

18 situation where identical-length paths differ in how they present risk. The risk cues are either a

19 live competitor (velvety tree ants, Liometopum occidentale (LO)) or formic acid (FA), a

20 defensive chemical commonly associated with formicine ant species. Across four paths to food,

21 the presence of cues also varied from always to never present. Although the non-risky path was

22 used more often, in no case did colonies completely avoid the paths with risk. Overall, more $L$.

23 humile workers explored paths associated with LO than with FA. This had a significant impact

24 on foraging ability where LO colonies were faster at finding food than FA colonies. Further, L.

25 humile workers' response to FA was similar over time while declined for LO, suggesting a 'dear

26 enemy' habituation and reduction in aggression over time. Thus, it appears that L. humile

27 foragers categorize risk cues and will vary their responses in potentially effective ways. 


\section{$\underline{\text { Introduction }}$}

Efficient food acquisition challenges all animals. Foragers navigate complex

33 environments that present a variety of costs and benefits, often associated both with the

34 distribution or quality of food patches and with the travel associated to reach them. Patch

35 exploitation and movements between patches can be influenced by travel distance, presence of

36 competing species or cooperating conspecifics, food quality, and hunger levels (Alatalo and

37 Lundberg 1986; Anderson 1984; Beckers et al. 1990; Conradt and Roper 2005; Vittori et al.

38 2006; Ronconi and Burger 2011; Yates and Nonacs 2016; Yamada 2017). Given that a forager's

39 first goal is to effectively encounter potential food items, the time it takes to discover food has

40 implications for foraging success and efficiency (Beverly 2009). In heterogeneous environments,

41 organisms may need to change their search strategies in order to efficiently find food. For

42 example, Argentine ants (Linepithema humile) appear to prioritize rapid recruitment to food once

43 it is found, rather than maximizing food encounter rates when search areas differ in spatial

44 complexity and food appears ephemerally (Denton and Nonacs, 2018).

Foraging strategies and spatial distributions of workers can also vary in response to the

46 risk that is present along a path. Lasius pallatarsis ant colonies abandon patches with associated

47 mortality risk to forage at "safer" patches (Nonacs and Dill, 1988). In Formica ants (F. perpilosa

48 and F. integroides), smaller foragers avoid sites at which risk is present and larger foragers may

49 spend more time at these sites in a defensive posture (Kay and Rissing, 2005; Tanner, 2008).

50 Maximizing foraging effort therefore requires recognizing key properties of different patches and

51 allocating foraging efforts across patches in a manner that increases time spent at "good" patches 
53 and decreases time spent at "bad." In heterogenous or changing landscapes, animals may then

54 need to sample their environment and develop expectations about future encounters to efficiently

55 exploit the area (Stephens and Krebs 1986). This process of combining older information with

56 newer information to alter expectations is known as Bayesian updating (Valone, 2006) and has

57 been observed in a wide variety of taxonomic groups (e.g. Lima 1984, 1985; Valone \& Brown

58 1989; Valone 1991, 1992; Krebs \& Inman 1992; Alonso et al. 1995; Olsson et al. 1999; van Gils

59 et al. 2003; Stamps et al. 2018).

Cooperatively foraging species, such as ants, add two more aspects to foraging strategies.

61 First, individual workers are 'disposable' in the sense that their deaths are expected and do not

62 directly influence the reproduction of the colony. Such foragers gain indirect fitness by helping

63 kin reproduce and may behave very differently from animals that risk their own reproductive

64 success (Nonacs and Dill, 1990). Second, many ant species mark and maintain trails that can

65 denote the optimal route to a food source; balancing trade-offs between various qualities amongst trails (Nonacs and Dill, 1990,1991, Latty et al. 2017).

In this study, we examine the path choice that ants make over time when: (1) There are

68 multiple paths of equal length to a food source, and (2) negative stimuli (i.e., cues that a

69 competing species may be nearby) are present along these paths in different frequencies, ranging

70 from $0 \%$ (never present) to $100 \%$ (always present). Previous studies have focused on decisions

71 between food and risk that are presented simultaneously, but here we focus on how colonies

72 learn and respond over time to negative features of their environment that may vary in how often

73 and predictably they are present. 
$\underline{\text { Methods }}$

79 Collection

We setup six replicate colonies of $L$. humile containing approximately 500-800 workers,

81 6-8 queens, and brood collected at the University of California, Los Angeles. The ants nested in

82 open plastic containers, filled with molded plaster of Paris, which was kept moist for nest

83 humidity, and coated on the sides with Fluon. Water was provided ad libitum.

\section{Experiment}

The experimental arenas consisted of four equidistant paths made of clear, plastic tubing

86 to a single foraging arena (distance $=90 \mathrm{~cm}$ ). To measure the time it took ants to find food, we

87 added sugar water to the foraging arena daily between approximately 12:00 and 15:00 h. Time

88 was recorded as how long it took the first ant to find the food and was recorded up to the first 10

89 minutes. Food was removed two hours after placement (whether or not ants were foraging) to

90 avoid colonies becoming satiated. To present the ants with negative stimuli, we placed cells at

91 the midway point of each path where cues were added (Fig. 1). Workers from three colonies each

92 encountered either several workers of the aggressive velvety tree ant (Liometopum occidentale

93 (LO)) (Hoey-Chamberlain et al. 2013) or formic acid (FA) on the paths and depending on the

94 treatment. LO and FA were placed along paths between 8:00 and 11:00 h. daily. They were

95 present along paths for approximately 24 hours and not correlated with the appearance of food.

96 The four paths differed such that LO or FA were present $0,25,50$ or $100 \%$ of trials. Therefore,

97 to reach food the L. humile foragers always had one path that never had risk

98 associated, another that always had risk, and two that might or might not have had risk associated

99 with them. This means that for any given trial, 1-3 paths could have had LO or FA present. 
We collected LO from Descanso Gardens (Los Angeles, CA) and housed them in a

101 similar manner to that of L. humile. Both L. humile and L. occidentale are in the same subfamily

102 of Dolichoderinae. FA is the primary defensive chemical used by ant species in the subfamily

103 Formicinae (Hefetz \& Blum 1978; Blum 1978). Colonies of ants were counted every four to five

104 days to ensure numbers did not drop drastically and workers were added if numbers dropped

105 below 500. Trials lasted approximately 20 days to allow each colony to experience all

106 frequencies of negative stimuli at least four times.

107 Risk cue: LO

108 We placed approximately 15-20 LO workers in cells 1-4 (Fig. 1). Mesh was present

109 between the LO workers and the L. humile workers, to prevent physical contact (Fig. S2).

110 However, they were able to exchange chemical cues. LO workers were removed and replaced

111 daily to ensure their numbers and effect were consistent over the course of the experiment.

$112 \quad$ Risk cue: FA

113 We placed cotton pads with $100 \mu \mathrm{L}$ of FA (Walmart, USA) in cells 1-4 (Fig. 1). We

114 covered the cells with lids for these trials to prevent the FA from completely dissipating over

115 time but cut holes (diameter $=2.54 \mathrm{~cm}$ ) into the encounter chambers for aeration and to prevent

116 FA fumes from accumulating in deadly amounts. We removed pads and/or replaced them daily

117 depending on the presentation schedule. If FA was to be absent for the day, we cleaned cells to

118 remove any leftover traces.

119 Video Observations

120 We programmed webcams to take fifteen-minute recordings of path use, approximately

121 three times per day: one at least three hours prior to food presentation (morning observation), one

122 while food was present (afternoon observation), and one in the evening, at least three hours after 
123 food had been removed (evening observation). The number of observations across colonies

124 varied because they were not all set up simultaneously and some observations had to be

125 discarded due to ants escaping or the clarity of video taken. Observations were at minimum 3

126 hours apart and at approximately the same time daily. We later scored these videos to examine

127 path use over time.

$128 \quad$ Video Scoring

129

130

Only the number of ants crossing the shaded portion of the experimental grids (Fig. 1)

131 was recorded as this was most detectable across all cameras and colonies. To avoid double-

132 counting an ant on a path, we kept spatial track of a forager's location and did not count it more

133 than once when simply moving back and forth on the same path.

\section{$\underline{\text { Statistics }}$}

We conducted linear-mixed effect models to study the amount of time ants took to find

138 food across days. Stimulus type was included in the model as well, as L. humile workers could

139 respond differently to LO or FA affecting finding times. We began by generating a null model

140 containing colony as a random intercept, then iteratively incorporated fixed effects into the

141 model, in order to find which combination of fixed effects generated the lowest AIC value for

142 the model. We conducted an ANOVA to examine mean time to find food across days by

143 stimulus type (FA or LO), all in R version 3.5.2 (R Core Team 2018).

144 We also conducted an ANOVA to examine path use of ants per day averaged across the

145 number of observations per day (log-transformed) against risk cue (LO or FA), path traveled ( 0 ,

$14625,50$ or 100$)$, number of paths with risk cues on a given day (i.e. "danger," ranging from 1-3),

147 and day of the experiment. Not every colony was tested on every day and therefore for statistical 148 analysis, time was divided into quartiles of 6 days each. 


\section{$\underline{\text { Results }}$}

In the analysis examining time to find food across days, including stimulus type as a

151 fixed effect significantly improved model fit (Table 1). This indicates that type of stimulus

152 predicts the amount of time it takes ants to find food. Further, in the analysis examining mean

153 time to find food across days by stimulus type, food was found significantly faster in LO

154 treatments than in FA treatments (Fig. 2; p < 0.0001). This indicates that foraging behavior (i.e.

155 time to find food) is influenced by stimulus type.

156 In the analysis examining number of ants per day against risk cue, path traveled, danger

157 (in terms of number of paths with risk cues present on a given day), and day of the experiment,

158 danger had neither a significant main effect nor any significant interaction effects. Therefore, it

159 was dropped as a factor in the ANOVA. In regard to the other main effects, significantly more

160 ants were on the paths with LO as opposed to FA (Fig. $3 ; \mathrm{p}<0.0001, \mathrm{~F}=154.87, \mathrm{df}=1)$. The

161 frequency of risk appearing on the paths also significantly affected their use (Fig. $3 ; \mathrm{p}<$

$1620.0004, \mathrm{~F}=6.205, \mathrm{df}=3$ ), where there was a significant difference between the number of ants

163 using the 0 and $25 \%$ paths $(\mathrm{p}<0.0061)$; the 0 and $50 \%$ paths $(\mathrm{p}<0.0001)$; and the 50 and $100 \%$

164 paths $(\mathrm{p}<0.0061)$. Additionally, the day of the experiment had a significant affect, where the

165 overall number of ants declined over time (Fig. $3 ; \mathrm{p}<0.0001 ; \mathrm{F}=9.681 ; \mathrm{df}=3)$.

166 When examining the 2-way interactions, we found when FA is the stimulus the number

167 of ants declined as risk got more frequent. However, for LO, the consistently riskiest path

$168(100 \%)$ drew the most ants (Fig. 3; $\mathrm{p}<0.0002, \mathrm{~F}=6.639, \mathrm{df}=3)$. Further, the FA response was

169 similar over time, while the LO response consistently declined (Fig. 3; $p<0.0001, F=10.130$, df

$170=3$ ). Except for the $100 \%$ path, the number of ants declined over time (Fig. 3: $\mathrm{p}<0.0026 ; \mathrm{F}=$

$1712.872, \mathrm{df}=9$ ). The 3-way interaction between stimulus, path, and time was not significant. 


\section{Discussion}

Cooperatively foraging species are a model system to examine how individuals with

174 limited knowledge and cognitive capabilities can achieve complex goals such as navigating

175 complex environments. Patch exploitation and movements between patches can be influenced by

176 a variety of factors including presence of competing species. While a forager's goal is to

177 successfully find food items, it also has to balance safety along paths. In heterogenous

178 environments, organisms may need to change their search strategies in order to efficiently find

179 food as well stay safe. Previous studies have focused on decisions between food and risk that are

180 presented simultaneously, but here we focus on how colonies learn and respond to negative

181 features of their environment that may vary in how often and how predictably they are present.

183 the two types of cues about risk drew significantly different responses both in regard to foraging

184 behavior and path use. Ants patrol/defend areas more intensely when in the presence of live LO

185 workers than with only FA, resulting in more ants on paths with LO as compared to FA. We can

186 attribute the lower numbers on paths with FA due to the effectiveness of this chemical weapon.

187 FA as a defense in formicine species is more effective in conflicts with Argentine ants

188 than the defenses of the dolichoderine velvety tree ants. This has been noted in Nylanderia fulva

189 and other formicines where formic acid as a chemical weapon achieves competitive dominance

190 in combat with Solenopsis invicta (LeBrun et al. 2014). Thus, even the occasional presence of

191 FA may depress Argentine ant activity in that area or path. As FA is a particularly effective

192 chemical weapon in combat, learning which trails to use or avoid FA is an important aspect of

193 their foraging strategy. 
Further, the large numerical difference in response along paths with live LO workers as

195 compared to FA likely resulted in LO colonies finding food when it appeared significantly faster

196 than FA colonies. This suggests that food discovery rate by Argentine ants can be directly

197 affected by their responses to encountering different competitive species. Additionally, their

198 response to FA is similar over time while for $\mathrm{LO}$, their response declines over time. This

199 suggests that the lack of direct contact and fights results in habituation and a 'dear enemy'

200 reduction in aggression over time (Langen et al. 2000) as L. humile workers decline in their

201 response to LO over time.

Additionally, the predictability of the risk along paths also had a significant effect on path

203 use. The always safe $(0 \%)$ path is used more overall than the paths with risk. Further, the overall

204 number of ants on the paths declines over time, except for the never safe (100\%) path. This

205 suggests that ants can assess and respond to risk present along paths and they moderate their

206 responses to paths that are not consistently risky but maintain a more consistent presence when

207 in response to paths that are always risky.

208 This work demonstrates that Argentine ants are able to learn about their environment and

209 use this information to effectively navigate and efficiently exploit their environment. Further,

210 this work elucidates how individual ants (with local knowledge and the ability to interact) can

211 scale up to effective organizations that optimally achieve vital, but potentially competing,

212 objectives. This is due to the fact that learning at the colony level is often more effective than at

213 the individual level as workers can vary in age and experience, and colonies allow for

214 communication and an increase in numbers. These results are relevant to understanding how

215 groups coordinate and function in a wide variety of species. 
216 Table 1: Model comparison for time data across days. Model $\mathrm{m} 2$ was chosen based on AIC

217 value and comparison with other models using likelihood ratio tests.

\begin{tabular}{|c|c|c|c|c|c|}
\hline Model Name & AIC & Model Formula & Comparison & Results & $\mathrm{P}$ \\
\hline $\mathrm{m} 0$ & 268.7 & $\ln$ Time $\sim(1 \mid$ Colony $)$ & N/A & N/A & N/A \\
\hline $\mathrm{m} 1$ & 269.0 & ln Time $\sim$ Day $+(1 \mid$ Colony $)$ & $\mathrm{m} 1, \mathrm{~m} 0$ & not significant & 0.1919 \\
\hline $\mathrm{m} 2$ & 260.4 & $\begin{array}{l}\ln \text { Time } \sim \text { Day }+ \text { Stimulus }+ \\
(1 \mid \text { Colony })\end{array}$ & $\mathrm{m} 2, \mathrm{~m} 1$ & significant & 0.0011 \\
\hline
\end{tabular}


251 Figure 1. Diagram of the experimental grid. Cells labeled 1-4 indicated where negative stimuli

252 were placed. All paths were of equal length.

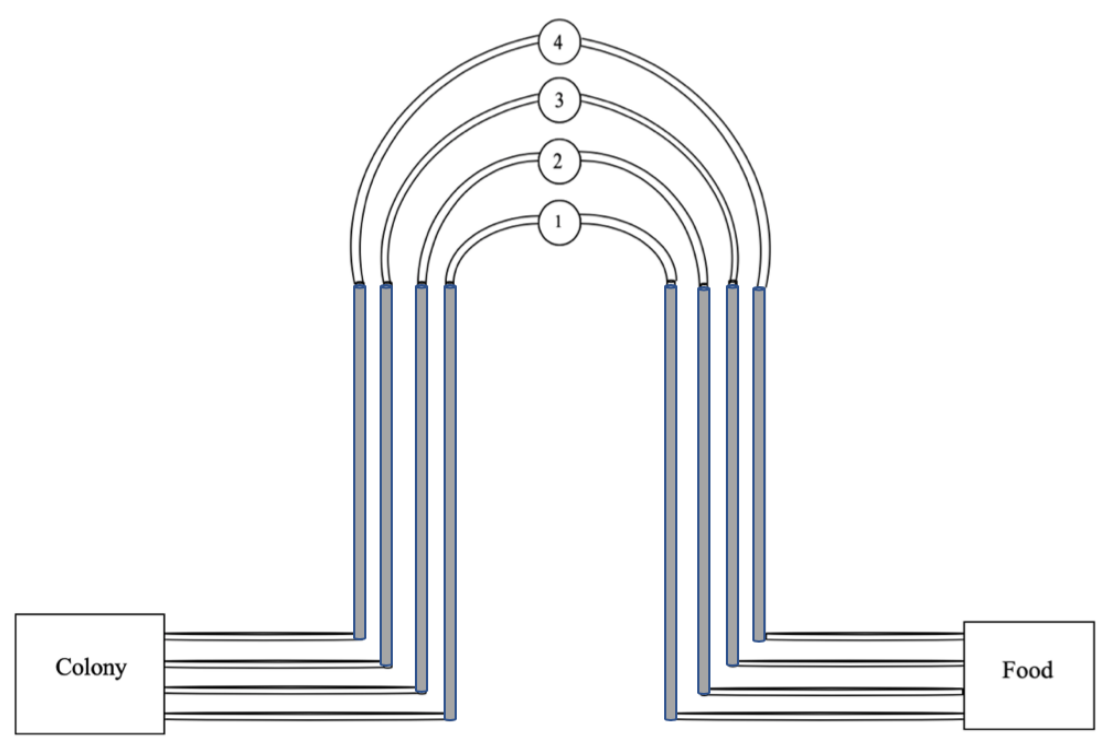


295 Figure 2: Mean time to find food across days for all four paths by stimulus type (where colony

296 1-3 received FA and 4-6 received LO). There was a significant difference in time to find food

297 between colonies that received FA as opposed to colonies that received LO $(p<0.0001)$.

298

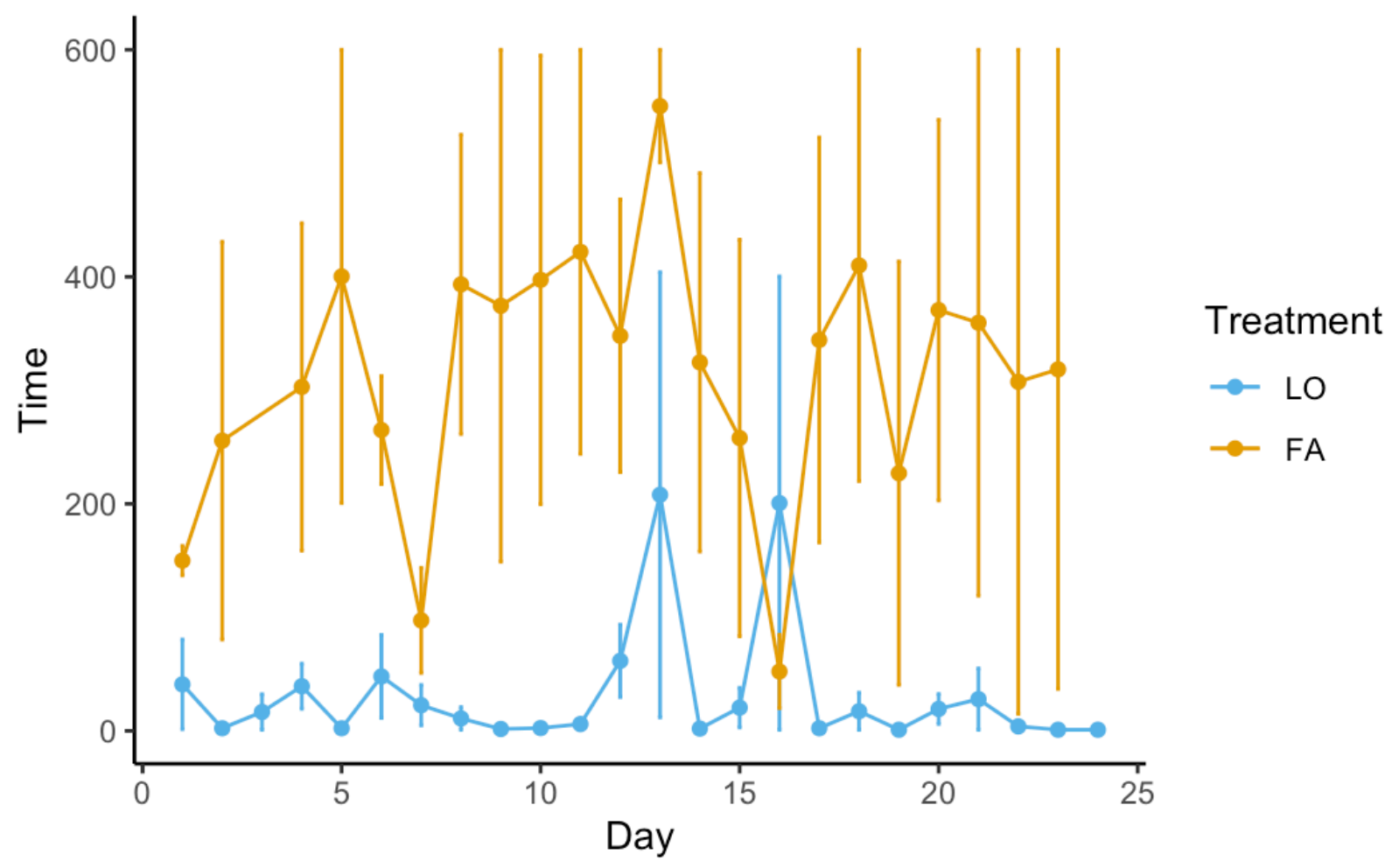


Figure 3: Mean number of ants per day averaged across the number of observations per day

309 (log-transformed) against risk cue (LO or FA), path traveled (0, 25, 50 or 100), number of paths

310 with risk cues on a given day (i.e. “danger," ranging from 1-3), and day of the experiment

311 (divided into quartiles). Colors respond to the division of days into 4 quartiles.

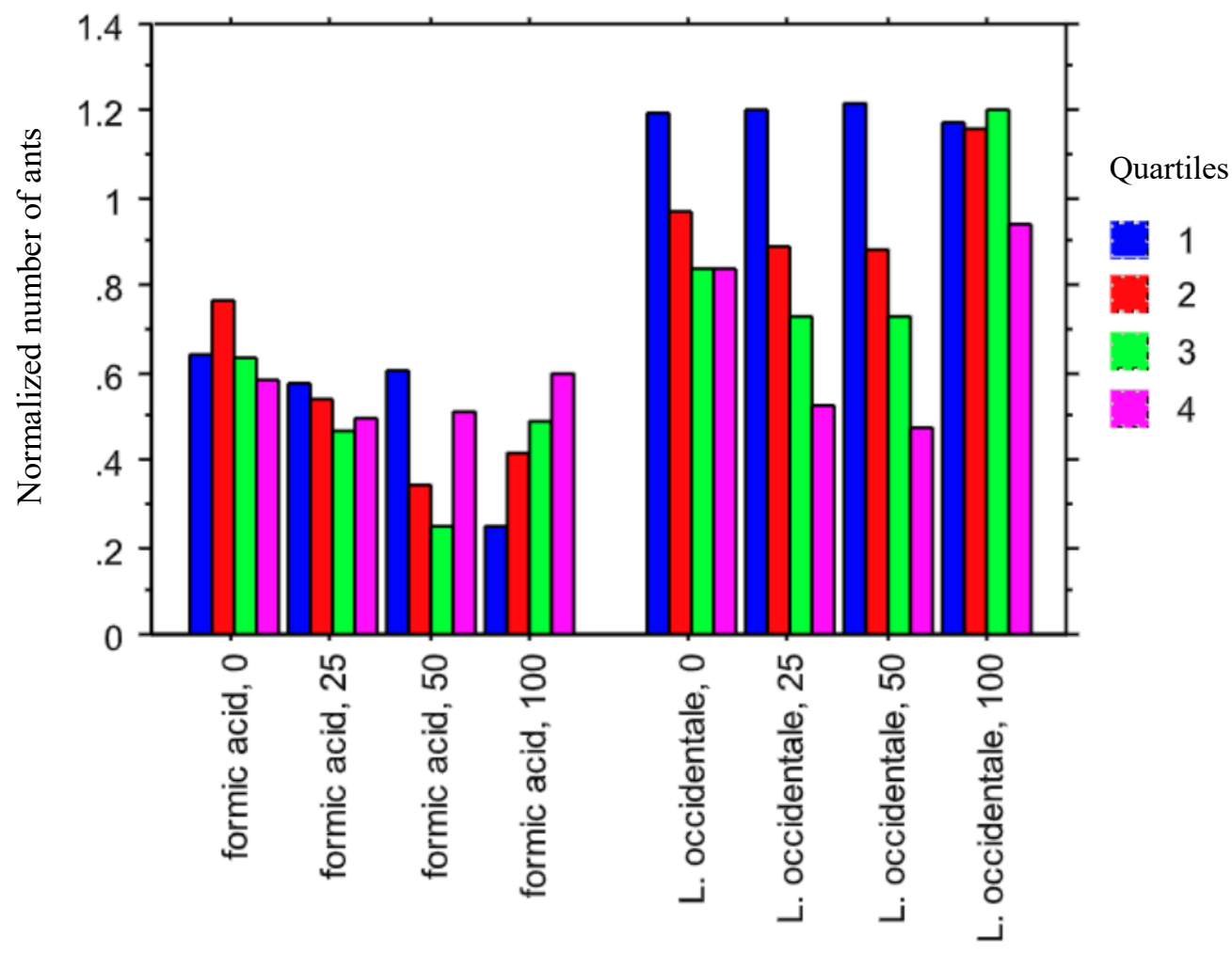

